\title{
The analysis of a prospective surgical database improves postoperative fast-tracking algorithms after pulmonary resection
}

\author{
Ayesha S. Bryant, MSPH, MD, ${ }^{\mathrm{a}}$ and Robert James Cerfolio, MD, FACS, FCCP
}

Objective: We evaluated our results from our prospective database to identify possible modifications that may improve our fast-tracking protocols in selected high-risk patients.

\begin{abstract}
Methods: We conducted a retrospective study of a prospective database. Using multivariable regression, we identified several patient characteristic that predicted failure to fast-track owing to increased morbidity. We modified our fast-tracking algorithm by substituting pain pumps for epidurals in elderly patients ( $>70$ years). In addition, patients with a body mass index greater than 35 had increased aspiration precautions. Patients with poor pulmonary function (ratio of forced expiratory volume in 1 second to forced vital capacity and/or diffusing capacity/alveolar volume $<45 \%$ ) underwent increased respiratory treatments and more aggressive ambulation. Differences in outcomes between groups were compared after adjusting for differing baseline patient characteristics, including use of a propensity score.
\end{abstract}

Results: A total of 2895 patients underwent elective pulmonary resection before the algorithm modifications (January 1997-December 2001) and 3252 patients afterward (January 2002-July 2007) by one surgeon. The length of stay was reduced by the protocol changes from 6.7 to 4.9 days $(P=.024)$ in elderly patients, from 5.7 to 4.8 days in obese patients, and from 6.2 to 4.3 days $(P=.008)$ in those with poor pulmonary function. Morbidity was reduced from $26 \%$ to $17 \%$ in elderly patients $(P=.046)$, from $29 \%$ to $20 \%(P=.027)$ in obese patients, and from $45 \%$ to $23 \%$ in those with poor pulmonary function. Overall mortality was also reduced $4.0 \%$ to $2.1 \%(P=.014)$.

Conclusion: A prospective database provides important information that can lead to improvement in patient care by identifying specific complications. High-risk patients such as the elderly, the obese, and those with poor pulmonary function can safely undergo pulmonary resection and have a shorter hospital stay.

The primary goals of any physician's care are to be safe and to maximize outcomes and patient satisfaction. However, we are all asked to achieve these results in an increasingly costconscience setting and in older patients who have more comorbidities than in the past. Fast-tracking protocols, also called postoperative computerized algorithms, allow for the consistent management of postoperative care after pulmonary resection. They have been shown to reduce cost and length of stay. ${ }^{1-4}$ These algorithms are especially helpful in a university or academic setting where the postoperative team often changes as residents and fellows rotate on and off the service. Prospective databases can provide powerful information about the success or failure of these post-

\footnotetext{
From the Department of Thoracic Surgery, Division of Cardiothoracic Surgery, University of Alabama at Birmingham, Birmingham, Ala. 'Assistant Professor, Division of Cardiothoracic Surgery, Department of Surgery, University of Alabama at Birmingham. ${ }^{b}$ Professor of Surgery, Chief of Section of Thoracic Surgery at University of Alabama at Birmingham Division of Cardio-Thoracic Surgery, Department of Surgery.

Received for publication May 6, 2008; revisions received Oct 28, 2008; accepted for publication Dec 21, 2008.

Address for reprints: Robert J. Cerfolio, MD, FACS, FCCP, Professor of Surgery, Chief of Thoracic Surgery, Division of Cardiothoracic Surgery, University of Alabama at Birmingham, $70319^{\text {th }}$ St S, ZRB 739, Birmingham, AL 35294 (E-mail: Robert.cerfolio@ccc.uab.edu).

J Thorac Cardiovasc Surg 2009;137:1173-9

$0022-5223 / \$ 36.00$

Copyright (c) 2009 by The American Association for Thoracic Surgery

doi:10.1016/j.jtcvs.2008.12.014
}

operative fast-tracking algorithms. Moreover, databases are frequently used by insurance companies and hospitals to asses the quality of care. Critical review of the results of these protocols can identify several groups of high-risk patients in whom fast-tracking is more likely to fail and complications are more likely to develop. The database allowed us to identify the most common types of complications that occur and in which types of patients. Modifications can then be implemented to allow for the reduction of these complications and afford safe fast-tracking of some of these groups of high-risk patients. The objective of this study was to compare the outcomes in several groups of high-risk patients both before and after changes had been implemented in our postoperative fast-tracking algorithms.

\section{METHODS}

This is a retrospective cohort study using a prospective database of a consecutive series of patients who underwent thoracotomy and elective pulmonary resection by one general thoracic surgeon in a university setting. Patients received similar postoperative care using fast-tracking protocols as previously described. The specifics of each postoperative day were outlined for the patient and their families. ${ }^{4}$ Two distinct time periods were used for this study. The first was from January 1997 until December 2001. The second was from January 2002 until July 2007. All patients in this study underwent thoracotomy, and if they had non-small cell lung cancer they also underwent complete thoracic lymphadenectomy..$^{5}$ Patients less than 19 years of age were excluded. Outcomes measured were hospital length of stay, transfer from the ward to the intensive care unit (ICU), overall and major morbidity, and operative mortality rates. The National Cancer Institute's 


\section{Abbreviations and Acronyms \\ $\mathrm{BMI}=$ body mass index \\ ICU $=$ intensive care unit}

booklet of adverse events was used for the definitions of the specific postoperative complications in this study. ${ }^{6-9}$ Operative mortality was defined as death for any reason before discharge or within 30 days of the operation. The Institutional Review Board at the University of Alabama at Birmingham approved the electronic prospective database used for this study as well as the study itself. Individual patient consent was obtained for entry into the prospective database, and patients were aware that these data would be used for research purposes. Individual consent for entry into this study was waived by the institutional review board.

\section{Specific Modifications to Fast-Tracking Protocols}

Using our data from January 1997 to December 2001, we identified the groups of patients most likely to have complications and also the most common and modifiable complication within each group (Table 1). Patient characteristics were studied as previously described. ${ }^{1}$ Those factors associated with failure to fast-track (complications that resulted in a greater hospital length of stay) were as follows: male gender, elderly age (elderly was defined as $>70$ years of age), history of neoadjuvant chemoradiotherapy, high body mass index (BMI) that we labeled obesity and that was defined as BMI greater than $35 \mathrm{~kg} / \mathrm{m}^{2}$ and poor pulmonary function status (poor pulmonary function defined as preoperative ratio of forced expiratory volume in 1 second to forced vital capacity $<45$ and/or diffusing capacity/alveolar volume $<45$ ), history of diabetes, and smoking. Using the findings of the multivariable analysis, we then selected several complications that we believed were the most modifiable and that occurred in the groups of patients with the highest rate of morbidity. The complications and the patient groups chosen were confusion and somnolence in elderly patients and pneumonia in obese patients and in those with poor pulmonary function.

We implemented specific changes in our postoperative algorithm in an attempt to reduce specific complications in the groups of high-risk patients during the second half of this study, which was from January 2002 until July 2007. Since somnolence and confusion were the main problems in elderly patients, we changed our protocol and stopped using epidurals and replaced them with intraoperative pain pumps. Somnolence was defined by the in-

TABLE 1. Univariate analysis showing the groups associated with having a morbidity and/or prolonged hospital stay

\begin{tabular}{|c|c|c|}
\hline & $\begin{array}{l}\text { Univariate analysis } \\
\text { outcomes for } \\
\text { morbidity or } \\
\text { mortality ( } P \text { value) }\end{array}$ & $\begin{array}{c}\text { Logistic regression } \\
\text { analysis outcomes for } \\
\text { complication } \\
\text { categories OR }(\mathrm{CI}), \\
P \text { value }\end{array}$ \\
\hline Age $>70 y^{*}$ & $<.001$ & $1.32(1.12-1.70), .043$ \\
\hline Gender* & .039 & $1.08(0.85-1.13), .215$ \\
\hline $\begin{array}{c}\mathrm{FEV}_{1 \%} \text { or } \mathrm{DLCO} / \\
\mathrm{VA}^{2} \%<45^{*}\end{array}$ & $<.001$ & $2.72(1.31-4.28),<.001$ \\
\hline Neoadjuvant therapy & .26 & - \\
\hline $\mathrm{BMI} \geq 35^{*}\left(\mathrm{~kg} / \mathrm{m}^{2}\right)$ & .010 & 1.45 (1.16-1.89), .026 \\
\hline Smoking history* & .015 & 1.29 (1.05-1.81), .044 \\
\hline $\begin{array}{l}\text { Lobectomy/ } \\
\text { bilobectomy }\end{array}$ & .64 & - \\
\hline
\end{tabular}

ability to arouse a patient without direct forceful external stimulus, which needed to be repeated more than once. Confusion was defined by the repeated inability of a patient to identify family members or to identify the correct setting or time of year. Since pneumonia and respiratory complications were the main problem in the obese patients and in those with poor pulmonary function, we asked for more respiratory therapists and nurses to help ambulate these patients. We presented these data to hospital administrators and in so doing received more physical and respiratory therapists, earmarked especially for these types of patients. This increased the number of respiratory treatments these patients received as well as the number of times they walked each day. The impact of these changes on patient outcomes is presented below.

\section{Statistics}

Univariate analysis was used to identify the groups of patients associated with complications. Continuous data are presented as medians and categorical data are presented as percentages. Fisher's exact test or the Pearson $\chi^{2}$ test was used to assess categorical data and the Wilcoxon test to evaluate continuous variables. Variables identified as having a possible relationship to outcomes $(P<.10)$ were entered into a backward stepwise multivariable regression model. Multiple logistic regression incorporating all significant baseline variables was used to derive a propensity score. Comparisons between groups regarding outcome variables were then performed unadjusted and adjusted for significant baseline variables and propensity score in multivariable regression analysis. All statistical analyses were performed with SAS software 9.0 (SAS Corporation, Cary, NC).

\section{RESULTS}

A total of 2895 patients underwent thoracotomy and elective pulmonary resection before the algorithm modification (January 1997-December 2001) and 3252 patients underwent surgery after the changes were implemented (January 2002-July 2007). Table 1 shows the univariate and multivariate findings for this study. It shows that age greater than or equal to 70 years, a ratio of forced expiratory volume in 1 second to forced vital capacity of less than $45 \%$, BMI greater than $35 \mathrm{~kg} / \mathrm{m}^{2}$, and smoking history were all predictors of morbidity.

Table 2 compares the patients' characteristics during the two times period of this study. The post-modification group had significantly more patients with BMI greater than $35 \mathrm{~kg} /$ $\mathrm{m}^{2}(P=.036)$ and patients with poor pulmonary function $(P>.001)$. These same factors independently differentiated these pre-modification and post-modification groups in stepwise multiple logistic regression analysis. These factors, together with missing value flags, were entered in multiple logistic regression analysis to create the propensity score. Table 3 compares the overall outcomes between the two groups and depicts the statistically significant lower morbidity and mortality in the post-modification group. When the groups were compared after propensity adjustment, the post-modification group had significantly shorter hospital length of stay $(P=.020)$ and incidence of morbidity $(P=$ $.004)$.

Table 4 shows the change in results in the elderly patients alone. It shows that the earlier group of patients were more likely to have a complication than the later group ( $26 \%$ vs 
TABLE 2. Patient characteristics both before and after the protocol changes

\begin{tabular}{|c|c|c|c|}
\hline & $\begin{array}{c}\text { Before protocol } \\
\text { change 1/1997-12/ } \\
2001 \\
\end{array}$ & $\begin{array}{c}\text { After protocol } \\
\text { change 1/2002-7/ } \\
2007\end{array}$ & $P$ value \\
\hline No. of patients & 2,895 & 3,252 & \\
\hline Gender (male) & $1852(64 \%)$ & $2016(62 \%)$ & .16 \\
\hline $\begin{array}{l}\text { Median age, y } \\
\quad \text { (range) }\end{array}$ & $67(19-86)$ & $68(19-85)$ & .21 \\
\hline $\begin{array}{l}\text { Obese patients } \\
\qquad(>35 \mathrm{BMI})\end{array}$ & $86(3 \%)$ & $130(4 \%)$ & .036 \\
\hline Median BMI & 42.1 & 43.8 & \\
\hline $\begin{array}{l}\text { Elderly patients } \\
\qquad(>70 \mathrm{y})\end{array}$ & $237(8 \%)$ & $277(9 \%)$ & .67 \\
\hline Median age (y) & 76.3 & 77.8 & \\
\hline $\begin{array}{l}\text { Poor pulmonary } \\
\text { function }\end{array}$ & $347(12 \%)$ & $520(16 \%)$ & $<.001$ \\
\hline
\end{tabular}

$17 \% ; P=.026)$. Table 5 depicts the outcomes for the obese patients. It shows a reduction in the overall morbidity and mortality as well as a reduction in the rate of pneumonia after the protocol changes was made. Finally, Table 6 shows the results for the patients with poor pulmonary function. It depicts a reduction in the incidence of pneumonia after respiratory therapy and ambulation were increased in the second half of this study.

\section{DISCUSSION}

The cost of health care continues to skyrocket and thus fast-tracking protocols that allow for high-quality care with reduced hospital stay and reduction in morbidity are more important today than ever before. Our fast-tracking algorithms implemented in 2001 employed the use of early water seal, removal of epidural catheters on postoperative day 2, early chest tube management using water seal, aggressive pulmonary toilet, and the treatment of persistent air leaks. Perhaps one of the most important caveats is the daily communication of the planned events for each postoperative day as well as the day of discharge with the family and pa-

TABLE 3. The overall outcomes between the two groups

\begin{tabular}{|c|c|c|c|c|}
\hline & $\begin{array}{c}\text { Before } \\
\text { modification } \\
(\mathrm{n}=\mathbf{2 8 9 5})\end{array}$ & $\begin{array}{c}\text { After } \\
\text { modifications } \\
(\mathbf{n}=\mathbf{3 2 5 2})\end{array}$ & $\begin{array}{c}\text { Unadjusted } \\
P \text { value }\end{array}$ & $P$ value* \\
\hline LOS (d) & 4.5 & 4.2 & .073 & .020 \\
\hline Morbidity & $926(32 \%)$ & $845(26 \%)$ & $<.001$ & .004 \\
\hline Mortality & 4.0 & 2.1 & .014 & \\
\hline $\begin{array}{c}\text { Mean operative } \\
\text { times (for } \\
\text { lobectomy) }\end{array}$ & $83 \mathrm{~min}$ & $72 \mathrm{~min}$ & .085 & .062 \\
\hline
\end{tabular}

TABLE 4. Outcomes in the high-risk elderly ( $>70$ years of age) patients

\begin{tabular}{lccr}
\hline & $\begin{array}{c}\text { Before } \\
\text { modification } \\
(\mathbf{n}=\mathbf{2 3 7})\end{array}$ & $\begin{array}{c}\text { After } \\
\text { modifications } \\
(\mathbf{n}=\mathbf{2 7 7})\end{array}$ & $\boldsymbol{P}$ value \\
\hline LOS (d) & 6.7 & 4.9 & .024 \\
Lobectomy & $165(70 \%)$ & $202(73 \%)$ & .764 \\
Segmentectomy & $47(20 \%)$ & $63(23 \%)$ & \\
Wedge & $18(8 \%)$ & $9(3 \%)$ & \\
Pneumonectomy & $1(1 \%)$ & 1 & \\
Other thoracotomy & $6(3 \%)$ & 2 & .026 \\
Morbidity & $62(26 \%)$ & $47(17 \%)$ & \\
$\quad$ Somnolence & 27 & 9 & \\
$\quad$ and/or & & & \\
$\quad$ confusion & & 38 & \\
$\quad$ Other & 35 & $3(1.1 \%)$ & .841 \\
Mortality & $4(1.7 \%)$ & & \\
\hline LOS, Length of stay. & &
\end{tabular}

tient. However, despite our efforts, our attempts to fast-track appeared to consistently fail in certain groups of patients. Even more concerning was that these groups of patients had complications. Although we could predict what the most common complication might be for some of these high-risk patients, we still were not always able to prevent it. Our prospective database allowed us to provide accurate data to hospital administrators and to administrative nursing services, which enabled us to implement changes in our postoperative algorithms and, even more important, justified the added cost of hiring additional personnel

Hospital readmission or recidivism rates are another important concern. If patients are sent home on the third postoperative day but are then in the emergency department 3 days later and are readmitted into the hospital, the cost savings of early discharge are negated. Furthermore, patient satisfaction plummets. The data for readmission rate in this series are not complete enough to accurately present.

TABLE 5. Outcomes in the high-risk obese $(B M I>35)$ patients before and after the protocol changes were implemented

\begin{tabular}{lccc}
\hline & $\begin{array}{c}\text { Before modification } \\
(\mathbf{n}=\mathbf{8 6})\end{array}$ & $\begin{array}{c}\text { After modifications } \\
(\mathbf{n}=\mathbf{1 3 0})\end{array}$ & $\boldsymbol{P}$ value \\
\hline LOS $(\mathrm{d})$ & 5.7 & 4.8 & .521 \\
Lobectomy* & $58(67 \%)$ & $96(73 \%)$ & .386 \\
Segmentectomy & $14(16 \%)$ & $15(12 \%)$ & \\
Wedge & 7 & 11 & \\
Pneumonectomy & 1 & 1 & \\
Other thoracotomy & 6 & 7 & .027 \\
Morbidity* & $25(29 \%)$ & $26(20 \%)$ \\
$\quad$ Pneumonia & 12 & 5 & .051 \\
$\quad$ All other & 13 & 21 & .718 \\
Mortality & $2(2.3 \%)$ & $1(0.8 \%)$ & \\
$L O S$, Length of stay. $* P$ value adjusted for significant baseline values and propensity \\
score.
\end{tabular}


TABLE 6. Outcomes in patients with poor pulmonary function $\left(\mathrm{FEV}_{1}\right.$ $<45 \%$ and/or DLCO/VA $<45 \%$ )

\begin{tabular}{lccr}
\hline & $\begin{array}{c}\text { Poor PFTs before } \\
\text { modification } \\
(\mathbf{n}=\mathbf{3 4 7})\end{array}$ & $\begin{array}{c}\text { Poor PFTs after } \\
\text { modifications } \\
(\mathbf{n}=\mathbf{5 2 0})\end{array}$ & P value \\
\hline LOS (d) & 6.2 & 4.3 & .008 \\
Lobectomy & $230(66 \%)$ & $405(78 \%)$ & $<.001$ \\
Segmentectomy & $59(17 \%)$ & $50(9 \%)$ & \\
Wedge & 34 & 42 & \\
Pneumonectomy & 1 & 1 & \\
Other thoracotomy & 23 & 22 & \\
Morbidity & $156(45 \%)$ & $120(23 \%)$ & $<.001$ \\
$\quad$ Pneumonia & 92 & 33 & $<.001$ \\
$\quad$ All other & 64 & 86 & .75 \\
Mortality & $5(2 \%)$ & $5(1.3 \%)$ & \\
\hline
\end{tabular}

$F E V_{l}$, Forced expiratory volume in 1 second; $D L C O / V A$, diffusing capacity for carbon monoxide/alveolar volume; PFTs, pulmonary function tests; $L O S$, length of stay.

This is partly due to the large geographic area that many of our patients come from. However, as we have more recently started to track recidivism, we have noted a significant number of patients $(4 \%-6 \%)$ are readmitted to home hospitals within 2 months of discharge, most commonly for dehydration, nausea, and what is often labeled pneumonia. Further studies and prospectively collected data are needed in this area.

In this article, we have shown several important concepts. First is that the accurate collection of data is powerful, not just for the construction of national databases for insurance companies or as political and reimbursement tools, but also for individual practices and patient care. Second, the careful and honest review of surgical results can identify recurrent complications that seem to occur in specific types of high-risk patients. Third, precise changes to postoperative protocols may be able to improve outcomes and their effect may be measurable.

This study has demonstrated that if epidurals are avoided in patients older than 70 years of age, confusion is less common. Elderly patients have less pain than younger patients and do not require epidural analgesia after thoracotomy if a rib-sparing, nerve-sparing technique is used, as $\mathrm{we}^{10,11}$ have previously described. Elderly patients are more sensitive to medications and their side-effects last longer owing to slower clearance of the drug by the kidneys or liver. Most elderly patients require only oral agents or even just acetaminophen 1 or 2 days after surgery. Second, patients who are obese or who have poor pulmonary reserve, who are known to be at increased risk after elective pulmonary resection, can have improved results and shortened hospital stays just by making changes in their postoperative care. This study shows that the investment made by the hospital by providing more ancillary services and by hiring more respiratory and physical therapists is a wise one and is profitable. We have shown that this simple step reduces the incidence of pneumonia and other complications. Patients receive better care and obtain better results.

As described in the Methods section, analysis of our prospective database allowed us to identify the groups at the highest risk and the most common complications within those groups. Specific modifications were then implemented for each of these groups. For the elderly patients, we avoided the use of epidurals. For obese patients and for those with poor pulmonary functions, we ordered aggressive physical therapy, pulmonary toilet, and mobilization regimens. Postoperatively, we increased the frequency of respiratory treatments that were actually being performed 6 times per day, not just ordered to occur 6 times a day. These simple but key modifications resulted in a decrease of hospital length of stay and morbidity rates in all three groups.

The strengths of this study include the high volume of patients and the fact that all procedures were performed by one surgeon. We did not knowingly alter our preoperative protocols over this time, and operative time, which decreased somewhat over the period of the study (72 compared with 83 minutes; $P=.085$ ), is an unlikely cause of the improved results. The limitations to this study, however, are obvious and several-fold. First, it is a retrospective study and is uni-institutional. Second, patients were not randomized. In most studies that compare two groups of patients over time, the second group often performs better than the first, especially when a team is used. There are several possible reasons for this phenomenon: the greater experience of the surgeon, who may have improved patient selection; a more experienced surgical team, which performs the same operations better or faster over time; and, most important, a more experienced postoperative team. Third, over each of the two time frames we have performed several prospective randomized studies; thus patients were in different groups during either the first half or the second half of the study. However, the number of patients in each group was relatively small (ie, lidocaine injection in the skin before thoracotomy in 66 patients compared with placebo injection in $53^{12}$; an intercostal muscle flap before chest retraction in 56 patients compared with no muscle flap in 58 patients ${ }^{10}$; and a nondivided muscle flap in 85 patients compared with a divided muscle flap in $75^{11}$ ). It is unlikely that these randomized studies that occurred within each time period blurred the overall differences detected between the two periods that featured 2895 patients in the first half and 3252 patients in the second half. Fourth, there may be other unrecognized factors that led to improved results in the latter group of patients. We have shown in Table 3 that there were fewer overall complications in the second group of patients in this study. However, when we eliminated the reduction in the lower incidence of somnolence and pneumonia, the statistical advantage was lost. This suggests the modifications were one of the main reasons for the improved results and not other unidentifiable reasons. Another limitation was 
the manner in which the three groups of patients were selected and the inherently subjective process of choosing the "most modifiable" complications in these groups. Finally, as shown in Table 2, there was a change in the distribution of these patient types over the two time periods. For example, we observed an increase in the incidence of elderly patients $(8 \%$ to $9 \%)$, an increase in the number of obese patients $(3 \%$ to $4 \%)$ and an increase in patients with compromised pulmonary function ( $12 \%$ to $16 \%$ ) during the second half of this study. These trends are not surprising and have been observed by others. ${ }^{13}$ These limitations must be considered when interpreting these data.

In summary, the construction of a prospective database and subsequent collection of accurate data allow for the review of surgical outcomes. The information can identify specific groups of high-risk patients who are more likely to incur specific types of complications. Outcome data provide the impetus to make changes to postoperative care algorithms, and these changes may improve surgical results.

\section{References}

1. Zehr KJ, Dawson PB, Yang SC. Standardized clinical care pathways for major thoracic cases reduce hospital cost. Ann Thorac Surg. 1998;66:914-9.

2. Bryant AS, Cerfolio RJ. The influence of preoperative risk stratification on fasttracking patients after pulmonary resection. Thorac Surg Clin. 2008;18:113-8.

3. Wright CD, Wain JC, Grillo HG, Moncure AC, Macaluso SM, Mathisen DJ. Pulmonary lobectomy patient care pathway: a model to control cost and maintain quality. Ann Thorac Surg. 1997;64:299-302.

4. Cerfolio RJ, Pickens A, Bass C, Katholi C. Fast-tracking pulmonary resections. J Thorac Cardiovasc Surg. 2001;122:318-24.

5. Cerfolio RJ, Bryant AS. Distribution and likelihood of lymph node metastasis based on the lobar location of non small-cell lung cancer. Ann Thorac Surg. 2006;81:1969-73; discussion 1973.

6. Bernard GR, Artigas A, Brigham KL, Carlet J, Falke K, Hudson L, et al. The American-European Consensus Conference on ARDS. Definitions, mechanisms, relevant outcomes, and clinical trial coordination. Am J Respir Crit Care Med. 1994;149:818-24

7. Schussler O, Alifano M, Dermine H, Strano S, Casetta A, Sepulveda S, et al. Postoperative pneumonia after major lung resection. Am J Respir Crit Care Med. 2006;173:1161-9.

8. Bryant AS, Cerfolio RJ. Differences in outcome between younger and older patients with non-small cell lung cancer. Differences in outcomes between younger and older patients with non-small cell lung cancer. Ann Thorac Surg. 2008;85: 1735-9.

9. National Cancer Institute. Common terminology criteria for adverse events. Version 3.0. New York: National Institutes of Health; 2003.

10. Cerfolio RJ, Bryant AS, Patel B, Bartolucci AA. Intercostal muscle flap reduces the pain of thoracotomy: a prospective randomized trial. $J$ Thorac of Cardiovasc Surg. 2005;130:987-93.

11. Cerfolio RJ, Bryant AS, Maniscalco L. A non-divided muscle flap further reduces the pain of thoracotomy. A prospective randomized trial. Ann Thorac Surg. 2008; 85:1901-6.

12. Cerfolio RJ, Bryant AS, Bass CS, Bartolucci AA. A prospective doubled blinded randomized trial evaluating the use of preemptive analgesia of the skin before thoracotomy. Ann Thorac Surg. 2003;76:1055-8.

13. Ferguson MK, Vigneswaran WT. Changes in patient presentation and outcomes for major lung resection over three decades. Eur J Card Surg. 2008;33:496-500.

\section{Discussion}

Dr K. Robert Shen (Rochester, Minn). Congratulations on an excellent presentation and study. Your group has been one of the strongest proponents of the idea that by critically analyzing and challenging many of the commonly used but unstudied aspects of the manner in which pulmonary resections are performed, important improvements and advances that benefit our patients can be achieved. We are all indebted to you for recent prospective randomized reports demonstrating that the use of preemptive analgesia of the skin before thoracotomy, the use of intracostal rather than paracostal sutures, and the use of an intercostal muscle flap all can reduce the pain of thoracotomy. Your group has also reported that the use of early water seal instead of suction on chest tubes and use of a classification system quantifying the magnitude of air leaks allow early dismissal from the hospital by allowing aggressive use of Heimlich valves as well as early chest tube removal in patients with prolonged air leaks or high outputs.

In this study, you have demonstrated, using a multivariate regression analysis, that several patient characteristics can be identified that can predict failure of your postoperative fast-tracking protocol owing to increased morbidity. The fast-tracking algorithm has been modified by substituting pain pumps for epidurals in elderly patients to decrease the incidence of postoperative confusion and oversedation and by the application of increased aspiration precautions and more frequent respiratory treatments and aggressive physical therapy in obese patients and those with marginal pulmonary function. This has resulted in a statistically significant reduction in the hospital length of stay, in morbidity, and in overall mortality.

Given all of the previously mentioned studies from your group during the study time period, it would seem to me that the conduct of the operation as well as many aspects of the postoperative care were very different for the patients in the contemporary study time period compared with those in the historical control. In the draft of the manuscript I received, you report a reduction in the mean operative time from 83 minutes to 72 minutes, a reduction of $14 \%$, which approached statistical significance, suggesting a learning curve effect. How can you be confident that the observed improvements in outcome were due to these specific modifications in the protocol and not simply the result of other modifications in your operative technique or postoperative care protocol that were implemented during this study period?

Dr Cerfolio. Thank you, Dr Shen, for your very kind comments. Your points are well taken. The short answer is, you are right. I totally agree. This is a fun study to shoot holes in. My 13-year-old, Alec, and my 10-year-old, Matthew, shot arrows at this one, and they and you should, because it is retrospective and flawed by all of the things that you mentioned. On the other hand, we have tried our best to be very specific about our changes, and many of our studies and changes probably did not affect this study's findings too much. I do not operate over Christmas and New Year's every year. I sit down with my team and say, "What did we do well this year and what did we do poorly?" We try to implement changes for the many things we did not do well starting in January. So there are other changes that occurred. You have mentioned some, and there are others that we know about and then probably some others that we are not aware of. We all know that any time you have a series, the second half of the patients you operate on do better than the first half because you are more experienced, your patient selection may get better, and your team is more experienced. Therefore, all of your points are correct. However, we tried and really could not identify anything more specific for the changes in our results than the ones we presented today. Finally, the 
operative time was not statistically significantly different because I have residents and fellows whom I am still teaching.

Dr Shen. One of the proposed benefits of fast-tracking protocols for surgical patients is that by standardizing postoperative care and adopting evidence-driven, best-demonstrated practices, there will be less variability in the care delivered. This will result in decreased hospital length of stay and a reduction in overall costs to the health care system, as well as a decrease in overall resource use. In the current environment of rising health care costs, this is an important goal. However, for real cost savings to be realized and resource use to be reduced, decreased initial hospital length of stay that results from adoption of fast-tracking protocols cannot be offset by higher rates of hospital readmission. My question is, do you know how the hospital readmission rates compare before and after these specific protocol modifications were implemented?

Dr Cerfolio. That is a great question. Although we do not have that specific number in the manuscript, it would be nice to know. I know now that our readmission rate is higher, but it is higher now because I actually have hired somebody to help us more honestly and specifically track that information. Now we do a better job getting that information and we have better follow-up. In addition, my 90-day mortality has gone up and my hospital readmissions have gone up now that I am actually more actively trying to find out what happens. It is ironic how many patients are not doing as well as they were when we saw them in the clinic at postoperative day 21 and we thought and reported that all was well.

Dr Shen. My final question is this: The modifications you have made to your fast-tracking protocol seem applicable to a wider group of patients than just those who are elderly, obese, and those with compromised pulmonary function. In your multivariate analysis, pneumonia was also the most common morbidity prolonging hospital stay in all of the other groups identified at higher risk for failure of a fast-tracking protocol, specifically those with male gender, white race, those undergoing induction therapy before resection, those with malignant etiologies, and adenocarcinoma histology. Have you considered applying the protocol modifications described here today to all of these subgroups in your clinical practice, and, if not, why not?

Dr Cerfolio. To answer your last question, what I always tell the residents and the fellows is that when dealing with hospital administrators, you try to win small battles one at a time and then maybe you will eventually win the war. I thought it would be unrealistic to ask to get enough respiratory therapists to treat all of our patients. We said: Here are the high-risk patients, the ones we are having morbidity with still. Here are our data of the patients who did poorly. Let's get enough ancillary assistance to take care of $20 \%$ or $30 \%$, which are our high-risk patients, and then we will show you data that shows the impact we have accomplished with that care. Then maybe with that information we will be able to get more help for the other patients as well. I did not know whether they would buy it up front for everyone, I agree that I want it for everybody.

Dr W. Roy Smythe (Temple, Tex). Will they buy a 1.8-day decrease in length of stay times 3000 patients, which is several million dollars of diagnosis-related group savings for the hospital?

Dr Cerfolio. I hope so, because we have shown them this abstract, and we look forward to its potential publication to do just that. We will see.
Dr Scott J. Swanson (New York, NY). Impressive numbers there, Dr Cerfolio. I have two quick questions. First, in the older than 70 group, do you know how many got lobeectomies, wedge resections, and segmentectomies, and did that change over the course of the 2 years?

Dr Cerfolio. I don not have that number, but I do not think it changed. I still favor lobectomies even for elderly patients. You and I know that whether they are 100 years old or whether they are 60 , it is their performance and pulmonary function, not their chronologic age, that matter. I would be surprised if there was a difference. I still favor lobectomy in the vast majority of patients.

Dr Swanson. Is that true for those with low pulmonary function as well?

Dr Cerfolio. No. Of course, if the patient had low pulmonary function, we would not favor lobectomy. I would do a segmentectomy for a lesion that is less than $2 \mathrm{~cm}$, but they all get the complete thoracic lymphadenectomy-all the N2 and N nodes come out.

Dr Swanson. Just to follow up on that last point, how do you gauge respiratory and physical therapists? Is it a certain number? Is it per patient? How do you make that argument? How many do you have?

Dr Cerfolio. That is a great question, and it varies on the day of the week, and, of course, it is different on the weekends. I used to try to only do 4 or 5 cases on Friday, and now I do 8 or 9 cases on Friday like my other operative days, on Monday, Tuesday and Wednesdays. Now only half of those are thoracotomies. I do not do more than 3 or 4 thoracotomies a day. You will see from my volume that half my cases are small, mediastinoscopies, video-assisted thoracic surgery, and so on, and half are thoracotomies. So it really varies. We try to do less risky procedures on Friday and maybe even fewer operations on Friday and try to gauge the amount of ancillary care per patient, per bed, per floor. That is how they tried to figure it out, but it seems social issues even dictate who we do and when, and then we do the best we can. We prefer 4 nurses per patient, and 1 respiratory therapist for 10 patients if possible.

Dr Mark J. Krasna (Towson, Md). I have a specific question regarding your patients who received chemotherapy and radiation therapy. Obviously during this time frame, you also increased the number of patients you were operating on after high-dose chemoradiotherapy.

Dr Cerfolio. Right.

Dr Krasna. Can you comment on the impact over the time frame in length of stay? Also, I know you are not trying to fast-track those patients, but, as Dr Shen mentioned, you are applying what you have learned now from those patients to those having postchemoradiotherapy lobectomy, for instance. How has that affected your length of stay and other outcomes?

Dr Cerfolio. That is a good question. One of the things that Dr Bryant mentioned is that the acuity has gone up. We have had more elderly patients in the second half, more patients with poor pulmonary function, and a lot more patients who got preoperative radiation and chemotherapy. We used to use $60 \mathrm{~Gy}$, then we went to 66 Gy, and now we are up to 72 Gy almost routinely. I have not really noticed a difference in the operative field because we are in there within a few months. I think fast-tracking is all about communication. If you tell the family in the clinic before surgery that the plan is this each day and that most go home on postoperative day 3 or 4 and you do them on Monday, and you tell them on Monday rounds, 
"Things went well in the operating room. You'll probably go home Wednesday or Thursday," and you tell them on Tuesday and you tell them on Wednesday, they go home Thursday because they are prepared for it mentally. It is all about communication and preparing things at home ahead of time. We have not seen an increase in length of stay. However, I think there is increased recidivism. We have seen more of those patients come back with just fatigue or weakness. They are billed as pneumonia by their home physicians when they get admitted, but when they get transferred back to us they seem to me many times to have a typical postthoracotomy radiograph; they are just weak. I think we have had a higher recidivism and readmission rate for those reasons, and maybe that is a failure of fast-track if they are more likely to come back.

Dr Michael T. Jaklitsch (Boston, Mass). The postoperative confusion, the delirium, the somnolence is really a continuous variable. How do you define that? Second, I am always interested in anybody who has ideas about how to avoid this in the over- 80 group. Do you adjust antidepressants or antianxiolytics? Do you adjust alcohol consumption preopoperatively? How do you manage the pain postoperatively?

Dr Cerfolio. Great questions. Your first question is about our definitions. We have very strict definitions in the manuscript for pneumonia and confusion, and these are all widely accepted. I can talk to you later about how we did it, but it is in the manuscript, and we were consistent throughout. That is one good thing about the study. One of the more difficult things about the study is that we did other things to decrease confusion. That sort of muddies our conclusions, as Dr Shen mentioned and I agree. I am not a fan of the ICU. When my patients go to the ICU, I lose control at the University of Alabama at Birmingham. They are being taken care of by another team and I cannot even write orders there. Often there is a junior resident who has not rotated on our service. Those residents and the ICU attendings are great, but I do not think they have the experience, nor do they have the reportability to me in the morning. We send almost nobody to the ICU, and that has helped decrease confusion. All 80-year-old patients having pneumonectomy go to the surgical ward. All patients having esophagectomy go to the surgical ward. They are monitored on the surgical ward, and it is a good surgical ward, but we avoid the ICU. This allows the family members to be around them all the time, and that really reduces confusion. An 82-year-old patient is not going to recognize me, but he or she is going to recognize a daughter. So we invite the family to be part of the care and stay in the room. That has helped us. We give them almost no drugs-almost nothing but acetaminophen (Tylenol) for pain.

Finally, your third question was about pain control. We insert these intraoperative subpleural pain pumps. We use those routinely when patients do not get an epidural. If they cannot get the epidural in place before the operation in 15 to 20 minutes, they are going to get a pain pump because we do not want to delay things. But none of the elderly patients get epidurals at all. When you drill holes in the bottom ribs, you do an intercostal nerve block; you do preemptive analgesia before you put the retractors in. Elderly patients have so much less pain than younger patients that all we give them, and this really works, is Tylenol, sometimes Extra-Strength Tylenol. If you can limit the medicines you give the elderly and give them almost nothing, they will not be confused. Their pain is not like that in a younger patient. We have even avoided ketorolac tromethamine (Toradol) in those patients because their creatinine levels come up high and we run them dry. Just Tylenol works well in most all of them. 